\title{
PHOTOIONIZATION OF EXCITED MOLECULAR STATES USING MULTIPHOTON EXCITATION TECHNIQUES *
}

\author{
P. M. Dehmer, S. T. Pratt, and J. L. Dehmer \\ Argonne National Laboratory, Argonne, Illinois 60439
}

\section{ABSTRACT}

Photoelectron spectra are reported for three photon resonant, four photon ionization of $\mathrm{H}_{2}$ via the $B{ }^{1} \Sigma_{L^{\prime}}^{+}, v=7 \quad(J=2,4)$ and $C^{1} \Pi_{u}$ ' $v=0-4 \quad(J=1)$ levels and of $N_{2}$ via the $o_{3}{ }^{1} I_{u}, \nabla=1,2, b{ }^{\prime}, v=3-5$, and $c{ }^{1} \mathrm{II}_{\mathrm{u}}$ v=0 levels. The results reflect both the spectroscopy and the dynamics of photoionization of excited molecular states and are discussed in terms of the selection rules for photoionization and the relative probabilities of photoionization from Rydberg and valence states. In some cases, in accordance with the Franck-condon principle, the results demonstrate that resonant multiphoton Ionization through Rydberg states may be a powerful technique for the production of electronic, vibrational, and rotational state selected ions. However, in other cases, systematic departures from Franck-Condon factors are observed, which reflect the more subtle dymamics of excited state photoionization.

\section{INTRODUCTION}

Resonantly enhanced multiphoton ionization (MPI) of molecules has been used to obtain detailed spectroscopic information on neutral intermediate states. 1 with the addition of photoelectron spectrometry (PES) to analyze the kinetic energy of the ejected electrons, it is possible to determine the branching ratios into different electronic, vibrational, and rotational levels of the product ion and to focus directly on both the dynamics of the multiphoton ionization process and the photoionization of excited state species. $2-6$ In an $(m+n)$ MPI/PES study, the excited state is prepared by m-photon excitation and subsequently is ionized by an additional n-photon excitation process. However, if $n$ is greater than 1, significant complications may arise in the photoelectron spectrum as a result of accidental resonances at higher intermediate levels. In the present paper, we report several $(3+1)$ MPI/PES studies of $\mathrm{H}_{2}$ and $\mathrm{N}_{2}$. These studies both illustrate the power of MPI/PES for the study of photoionization of excited molecular states and demonstrate the possibility of producing electronic, vibrational, and rotational state-selected ions using selective multiphoton ionization.

* Work supported by the U.S. Department of Energy and the Office of Naval Research. 
The apparatus for these studies consists of a commercial Na: YAG pumped dye laser systein, a 2-inch mean radius hemspherical electron energy analyzer, and a time-of-flight mass spectrometer. 3 In a typical experiment, the photoion spectrum is recorded as the wavelength of the dye laser is scanned. The laser is then tuned to the wavelength of a specific rovibronic transition and the photoelectron spectrum is recorded. In the measurements reported here, photoelectron spectra were recorded along the polarization axis of the laser with a photoelectron energy resolution of $\sim 35$ meV. For most of the experiments, the electron spectrometer was tuned so that the transmission function over the kinetic energy range of interest was nearly constant. The corrections for the transmission function are less than $20 \%$ between 0.5 and $1.6 \mathrm{eV}$ (electron kinetic energy), but may be substantial in the region belo: $0.5 \mathrm{eV} .3^{3}$ Under the conditions of the present experiments, nonresunant ionization was not observed.

\section{PHOTOIONIzATION OF THE B ${ }^{1} \Sigma_{u}^{+}$STATE OF $\mathrm{H}_{2}$}

Photoionization of the ${ }^{1} \Sigma_{\mathrm{u}}^{+}$state of $\mathrm{H}_{2}$ is presented as the initial example, 4 since it serves to illustrate the method of obtaining the selection rules for a $(3+1)$ multiphoton ionization process. The selection rules appropriate for three photon transitions from the ground electronic state to the ${ }^{1}{ }_{\Sigma_{u}^{+}}^{+}$state of $\mathrm{H}_{2}$ are summarized schematically in Figure 1. The three photon selection rules based on symmetry properties are identical to those for single photon transitions ( $g \leftrightarrow u,+\leftrightarrow-$, and $s \leftrightarrow-a) .7,8$ In addition, for a three photon transition, $\Delta J<3$. It is clear that the only transitions from the ground state to the intermediate $B{ }^{1}{ }_{u}^{+}$ state that fulfill all of the selection rules have $\Delta J= \pm 1, \pm 3$.

The structure in the photoelectron spectrum obtained by ionizing a specific rotational level of the $B{ }^{1} \Sigma_{u}^{+}$state is determined by the single photon transition from the intermediate level to the ionization continua. The selection rules governing this transition can be deduced by considering the $\mathrm{H}_{2}^{+}{ }_{7}^{\mathrm{X}}{ }_{\mathrm{I}}^{+}$ion and the ejected electron in Hund's case (d) coupling.7 Here, the excited electron is completely uncoupled from the internuclear axis, and the rotational angular momentum of the ion core $N$ and the orbital angular momentum of the ejected electron \& are added vectorially to give the total angular momentum $J$ of the electron-ion complex. Owing to the $g^{t+u}$ selection rule, the outgoing electron must have an even value of $l$. In the present work, only $s$ and $d$ partial waves are considered; higher partial waves will have a large centrifugal barrier and may be ignored. By considering the various selection rules for the ionizing transition, the allowed final rotational levels of the ion can be derived. It is seen from Figure 1 that the ionizing transition must obey the selection rule $N\left(X^{2} \Sigma_{g}^{+}\right)-J\left(B{ }^{1} \Sigma_{u}^{+}\right)= \pm 1, \pm 3$, and that for 1 given intermediate rotational level, the ion will be left in only odd or only even rotational states. Because the range of allowed $\mathrm{H}_{2}^{+}$rotational 


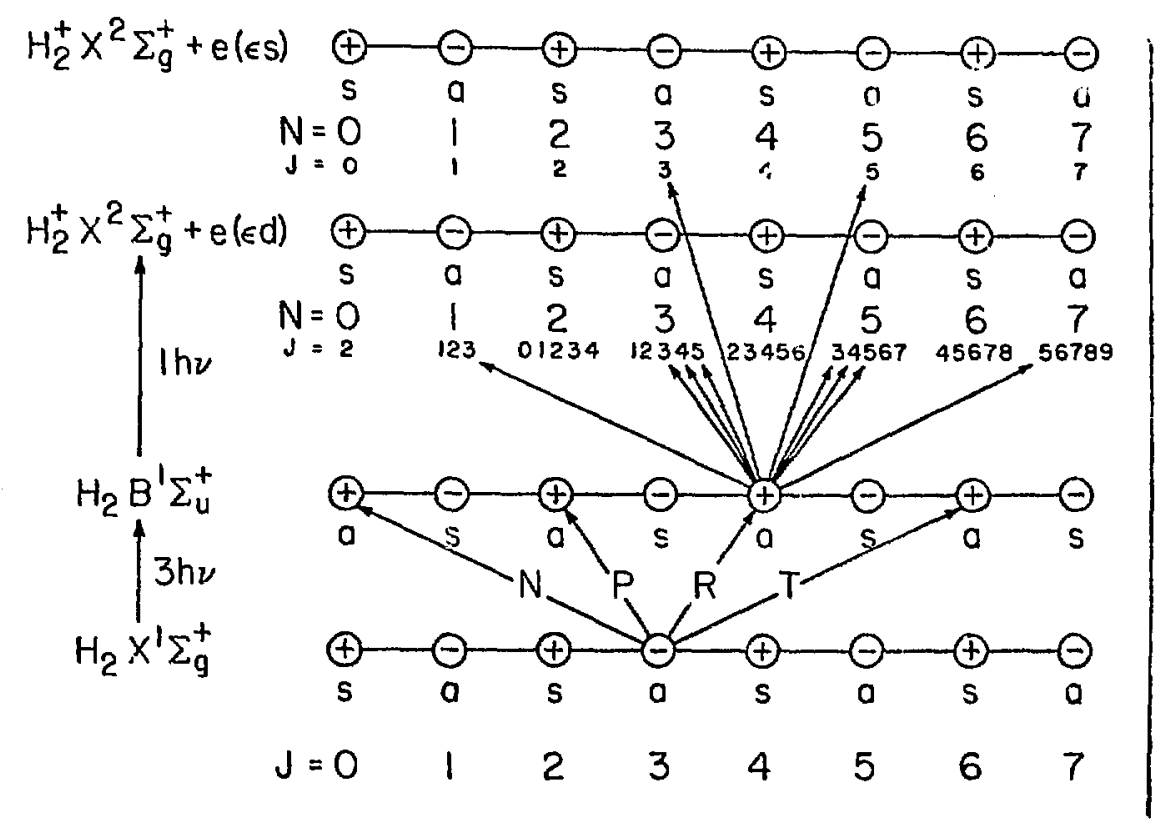

Figure 1. Schenatic diagram of the allowed transitions for the $(3+1)$ resonant multiphoton Ionization of $\mathrm{H}_{2}$ via the $B{ }^{1} \Sigma_{u}^{+}$state. Only those transitions from $J=3$ of the ground state and $J=4$ of the intermediate state are shown.

values differs for $s$ and $d$ waves, a rotationally resolved photoelectron spectrum can give detailed information on the relative importance of the different partial waves. We also note that in the angular momentum transfer $\left(j_{t}\right)$ formulation, $9-11$ the ejection of an $s$ electron can lead only to a $j_{t}$ value of 1 , while the ejection of a $d$ electron can yield $j_{t}$ values of 1,2 , and 3 . whe $j_{t}$ value of 2 is parity unfavored, and only values of 1 and 3 are important here.

Figure 2 shows the photoelectron spectra obtained by pumping the $R(3)$ and $P(3)$ transitions of the $B{ }^{1} \Sigma_{u^{\prime}}^{+} v^{\prime}=7+x^{\prime} \Sigma_{g^{\prime}}^{f} v^{*}=0$ band. The most dramatic feature of the photoelectron spectra is that the observed rotational structure changes qualitatively with the intermediate rotational level of the $\mathrm{B}^{1 \mathrm{~S}_{\mathrm{u}}^{+}}$state, thus directly reflecting the selection rules for the ionizing transition.

In the photoelectron spectrum obtained by pumping the $R(3)$ transition, the $\mathrm{N}=3$ and 5 rotational levels of the ion clearly are observed. Although it is allowed by selection rules, the $N=7$ level is not observed in any of the vibrational bands, indicating that the $\Delta \mathrm{J}=+1, \Delta \mathrm{N}=+3, j_{t}=3$ ionizing transition, which corresponds to the ejection of a d-wave electron, is weak. similarly, only the $N=1$ and 3 levels are observed in the photoelectron spectrum obtained by pumping the $P(3)$ transition, whereas the $N=5$ level (which again requires $\Delta J=+1, \Delta N=+3, j_{t}=3$ and the ejection of a d-wave electron) is not observed. In the photoelectron spectrum obtained by pumping 


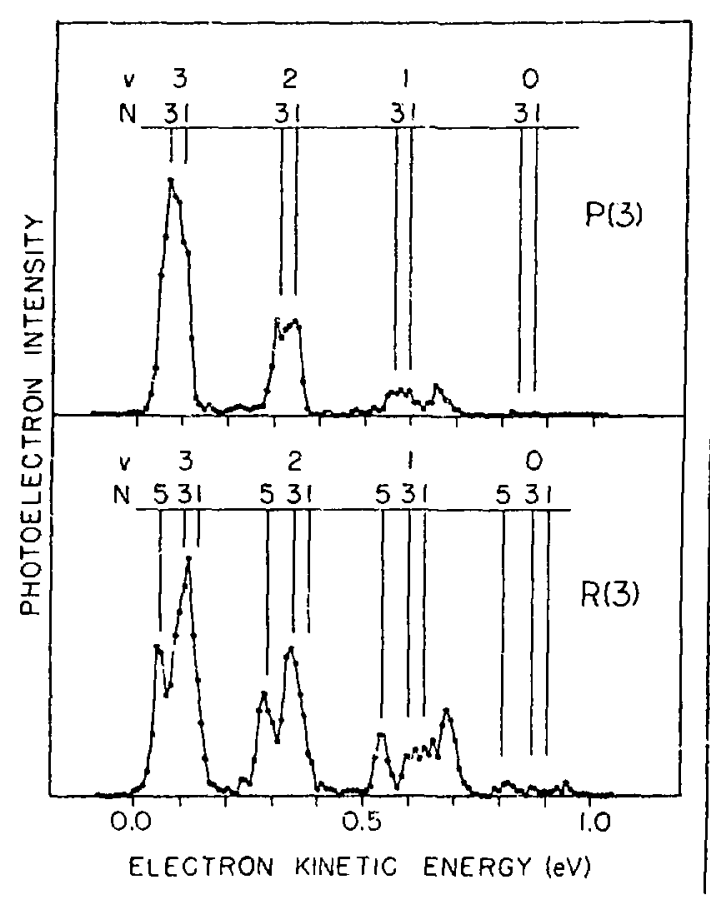

Figure 2. Photoelectron spectra of $\mathrm{H}_{2}$ determined following $(3+1)$ multiphoton ionization at the wavelengths of the resonant three photon $\mathrm{B}^{1} \Sigma_{\mathrm{u}^{\prime}}^{+}, \mathrm{v}^{\prime}=7$, $J^{\prime}=2,[P(3)]$ and $J^{\prime}=4 \quad[R(3)]$ $+\mathrm{X}^{1} \Sigma_{\mathrm{g}^{\prime}}^{+} \mathrm{v}^{\prime \prime}=0, \mathrm{~J}^{\prime \prime}=3$ transitions. The unassigned peaks at $0.69 \pm 0.01 \mathrm{ev}$ in the $R(3)$ spectrum and at $0.67 \pm 0.01$ in the $P(3)$ spectrum are believed to result from photoionization of $\mathrm{H}(2 \mathrm{~s}$ or $2 \mathrm{p})$ formed by photodissociation of $B^{1} \Sigma_{u}^{+}$.

the $R(3)$ transition, the $N=1$ rotational level of the ion appears to be missing. The $\mathrm{N}=1$ level is allowed by selection rules, but requires $\Delta J=-1, \Delta N=-3, j_{t}=3$, and the ejection of a d-wave electron. Together with the other evidence, this indicates that either $j_{t}$ values of 3 or the ejection of a d-wave electron are very weak in the photoionization processes studied here. of these two possibilities, it is most likely that $j_{t}$ values of 3 are weak in the photoinization process. Transitions of the type $2 \mathrm{po} \mathrm{B}^{7} \Sigma_{\mathrm{u}}^{+}+\mathrm{nd} \sigma$, nd $\pi$, nd $\delta$ have been observed in emission, 12,13 making it likely that the ejection of a d-vave electron will be observed in photoionization.

\section{PHOTOIONIZATION OF THE $C{ }^{1} \Pi_{u}$ STATE OF $\mathrm{H}_{2}$}

In this experiment, 5 photoelectron spectra were determined following single photon ionization of the $\mathrm{C}^{1} \pi_{\mathrm{u}^{\prime}}^{-} \mathrm{J}^{\prime}=1$ levels of $\mathrm{H}_{2}$ accessed by $Q(1)$ transitions from the ground state. The method of obtaining the selection rules for this process is identical to that for the case of ionization of the ${ }^{B}{ }^{1} \Sigma_{u}^{+}$state described above. In this case, it is found that the $\mathrm{H}_{2}^{+}{ }_{\mathrm{X}}{ }^{2} \Sigma_{\mathrm{g}}^{+}$ion can be left with rotational angular momentum $\mathrm{N}=1,3 .{ }^{2}$ The ${ }^{\mathrm{N}} \mathrm{N}=1$ and 3 levels are separated by $\sim 30 \mathrm{mev}$ (in $\mathrm{v}^{+}=3$ ) and will not be resolved with the present spectrometer resolution. The production of $\mathrm{H}_{2}^{+} \mathrm{X}^{2} \Sigma_{\mathrm{g}^{\prime}}^{+} \mathrm{N}=3$ requires an outgoing $d$-wave and an angular momentum transfer $j_{t}=3$. As is discussed above, such a process was not observed in the study of photoionization from the $\mathrm{B}^{1} \Sigma_{\mathrm{u}^{+}}^{+}$state. Thus it. is tempting to speculate that the $\mathrm{H}_{2}^{+}$ions result.ig from photoionization of the $c^{1} \pi^{-}, J^{\prime}=1$ levels are produced predominately in the $N=1$ level, 
i.e., the ions are produced in a single rotational level.

As in the case of single photon transitions, the $P$ and $R$ branches access the $\Pi^{+}$component of the ${ }^{\Lambda \text {-doubled } C}{ }^{1} \Pi_{u}$ state, while the $Q$ branch accesses the $\pi^{-}$component. 7 This is important for the present photoelectron studies, since the $v^{\prime}=0-4$ levels of the $c{ }^{1}$ state are perturbed by the $v^{\prime}=8,10,12,14,16$ levels of the $B{ }^{1} \Sigma_{u}^{+}$ state, respectively. ${ }^{14}$ Fortunately, the $c{ }^{1} \Pi_{u}^{-}$levels are unatfected by this perturbation. 7 Therefore, by pumping the Q-branch transitions, the effects of perturbations of the intermediate level on the vibrational branching ratios following multiphoton ionization are avoided.

The photoelectron spectra obtained at the wavelengths of the three photon $Q^{(1)}$ transitions of the $c^{1} I_{u^{\prime}} v^{\prime}=0-4+x^{1} \Sigma_{g^{\prime}}^{+} v^{n}=0$ bands are shown in Figuxe 3. The most striking aspect of the photoelectron spectra is the dominance of the photoelectron peak corresponding to the $v^{+}\left(x^{2} \Sigma_{g}^{+}\right)=v^{\prime}\left(c^{1} \Pi_{u}\right)$ transition. In addition, the weaker peaks with the greatest intensity are those adjacent to the $\mathbf{v}^{+}=v^{\prime}$ peak. This agrees with expectations based on FranckCondon factor calculations (Table I).15 However, while the qualitative agreement with the calculations is very good, the quantitative agreement is poor. For example, in the spectrum obtained via the $C^{1} I v, v^{\prime}=4$ level, the $v^{+}=3,5$, and 6 peaks are too large by factors of 3,2 , and 23 , respectively, and the intensity of the $v^{+}=4$ peak accounts for only 438 of the total, rather than the predicted $90 \%$.

Table I. Franck-Condon factors for $\mathrm{H}_{2}^{+} \mathrm{x}^{2} \mathrm{\Sigma}_{g^{\prime}}^{+} \mathrm{v}^{+}+\mathrm{H}_{2} \mathrm{C}^{1} \mathrm{I}_{\mathrm{u}^{\prime}} \mathrm{v}^{\prime}$

$v^{+} \quad v^{\prime}=0 \quad v^{\prime}=1 \quad v^{\prime}=2 \quad v^{\prime}=3 \quad v^{\prime}=4$

\begin{tabular}{rlllll}
\hline 0 & $9.89 \times 10^{-1}$ & $1.10 \times 10^{-2}$ & $4.67 \times 10^{-6}$ & $1.45 \times 10^{-8}$ & $2.26 \times 10^{-9}$ \\
1 & $1.06 \times 10^{-2}$ & $9.66 \times 10^{-1}$ & $2.29 \times 10^{-2}$ & $2.04 \times 10^{-5}$ & $2.98 \times 10^{-8}$ \\
2 & $3.08 \times 10^{-4}$ & $2.16 \times 10^{-2}$ & $9.43 \times 10^{-1}$ & $3.55 \times 10^{-2}$ & $6.47 \times 10^{-5}$ \\
3 & $1.46 \times 10^{-5}$ & $9.98 \times 10^{-4}$ & $3.22 \times 10^{-2}$ & $9.19 \times 10^{-1}$ & $4.80 \times 10^{-2}$ \\
4 & $1.04 \times 10^{-6}$ & $6.53 \times 10^{-5}$ & $2.13 \times 10^{-3}$ & $4.16 \times 10^{-2}$ & $8.96 \times 10^{-1}$ \\
5 & $1.00 \times 10^{-7}$ & $5.95 \times 10^{-6}$ & $1.82 \times 10^{-4}$ & $3.71 \times 10^{-3}$ & $4.92 \times 10^{-2}$ \\
6 & $1.22 \times 10^{-8}$ & $7.24 \times 10^{-7}$ & $2.03 \times 10^{-5}$ & $3.98 \times 10^{-4}$ & $5.69 \times 10^{-3}$ \\
7 & $1.87 \times 10^{-9}$ & $1.12 \times 10^{-7}$ & $2.92 \times 10^{-6}$ & $5.42 \times 10^{-5}$ & $7.43 \times 10^{-4}$ \\
8 & $3.77 \times 10^{-10}$ & $2.04 \times 10^{-8}$ & $5.34 \times 10^{-7}$ & $8.99 \times 10^{-6}$ & $1.21 \times 10^{-4}$ \\
9 & $1.04 \times 10^{-10}$ & $4.38 \times 10^{-9}$ & $1.19 \times 10^{-7}$ & $1.82 \times 10^{-6}$ & $2.43 \times 10^{-5}$ \\
10 & $3.75 \times 10^{-11}$ & $1.12 \times 10^{-9}$ & $3.02 \times 10^{-8}$ & $4.65 \times 10^{-7}$ & $5.68 \times 10^{-6}$ \\
\hline
\end{tabular}

The most likely causes of such deviations are: (1) a kinetic energy dependence of the electronic transition matrix element, which nust be taken into consideration even within the Franck-Condon approximation; (2) an R-dependence of the same electronic transition 
matrix element, which, by definition, constitutes a breakdown of the Franck-Condon approximation; (3) a $\mathbf{v}^{+}$-dependence of the photoelectron angular distribution; and (4) other effects such as perturbations or overlapping lines at the three photon level and accidental resonances with autoionizing states at the four photon level. of these possibilities, accidental resonances and perturbations are not important in the present case. 5 Hence, the data in Figure 3 represent a well defined case for further experimental and theoretical investigations of the excited state dynamics outlined in $(1)-(3)$ above.

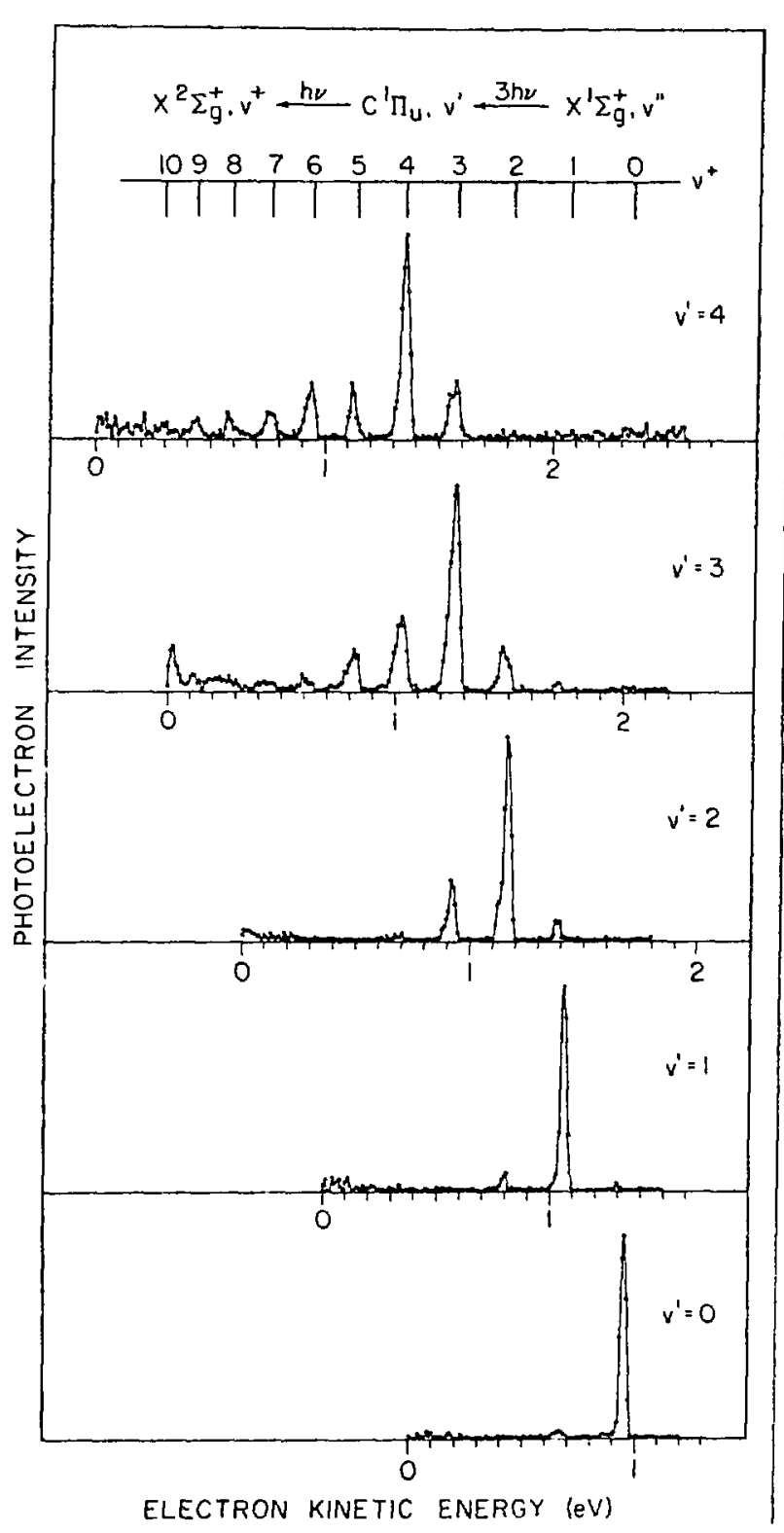

Figure 3. Photoelectron spectra of $\mathrm{H}_{2}$ determined following (3+1) multiphoton ionization at the wavelengths of the resonant three photon $C^{1} \pi_{\mathcal{u}^{\prime}}, \quad v^{\prime}=0-4+\mathrm{x}^{1} \Sigma_{\Sigma^{\prime}}^{+}$ $v^{\prime \prime}=0, \quad Q(1) \quad$ transitions. 
phOTOIONIZATION OF THE $o_{3}{ }^{1} \mathrm{I}_{u}$ STATE OF $\mathrm{N}_{2}$

This system serves to demonstrate the production of electronically excited, vibrational state-selected molecular ions by resonant multiphoton ionization via a Rydberg state with an electronically excited ion core. Specifically, it is shown that the dominant ionization pathway for the $(3+1)$ multiphoton ionization of $\mathrm{N}_{2}$ via the $\mathrm{o}_{3}{ }^{1} \Pi_{\mathrm{u}}, \mathrm{v}^{\prime}=1,2$ levels leads to the production of $\mathrm{N}_{2}^{+}$ $\mathrm{A}^{2} \pi_{u^{\prime}} \mathrm{y}^{+}=1,2$, respectively. 6 The ${ }^{\circ}{ }_{3}^{1} \mathrm{I}_{\mathrm{u}}$ state of $\mathrm{N}_{2}$ has the electron configuration ... $\left(\frac{1}{6} \pi_{\mathrm{u}}\right)^{3}\left(3 \sigma_{\mathrm{g}}\right)^{2} 35 \sigma_{\mathrm{g}}$ and is the lowest member of Worley's third series, 16 which converges to $\mathrm{s}_{2}^{+}{ }^{2} \Pi_{1 / 2}$. The ionizing transition strongly favors the removal of the outer $3 s \sigma_{g}$ electron leading to the direct production of $\mathrm{N}_{2}^{+} A{ }^{2} \Pi_{u^{*}}$. This is the first experimental evidence in a molecular system showing the degree to which the electronic excitation of the ion core is retained following photoionization of a Rydberg state. Similar effects have been observed recently in the photoionization of the rare gas atoms; $^{17}$ that is, in all of the observed transitions, the spin-orbit state of the ion core was preserved.

In addition to preserving the electronic state of the ion core, the $\mathrm{A}^{2} \pi_{\mathrm{u}}+\mathrm{o}_{3}{ }^{1} \pi_{\mathrm{u}}$ ionlzing transition also preserves the vibrational level of the $o_{3}{ }^{1} \pi$ Rydberg state. This is in accord with calculations for the $A^{3}{ }^{2} u_{u^{\prime}} v^{+}+0{ }^{1} \Pi_{u^{\prime}} v^{\prime}$ ionizing transition using Morse potentials for both states, which give Franck-Concon factors greater than 0.99 for the $\mathrm{v}^{+}=\mathrm{v}^{\prime}$ transition for $\mathrm{v}^{\prime}=1$ and 2 . However, as shown above in the case of the $C^{1} \pi_{u}$ state of $\mathrm{H}_{2}$, one, in general, cannot rely on the Franck-Condon principle to predict vibrational branching ratios for excited state photoionization.

Photoelectron spectra were recorded at the wavelengths corresponding to the three photon transitions to the R-branch bandhead of the $\mathrm{o}_{3}{ }^{1} \pi_{\mathrm{u}^{\prime}} \mathrm{v}^{\prime}=2+\mathrm{x}^{1} \Sigma_{\mathrm{g}^{\prime}}^{+} \mathrm{v}^{\prime \prime}=0$ band and to three positions, including the R-branch bandhead, with $n$ the $o_{3}{ }^{1} \Pi_{u}, v^{\prime}=1$ ${ }^{+} \mathrm{X}^{1} \Sigma_{\mathrm{g}}^{+}$, $\mathrm{v}^{n}=0$ band. Figure 4 gives the plucoelectron spectra obtained via the $o_{3}{ }^{1} I_{u^{\prime}} v^{\prime}=1$ and 2 bands. Of the three spectra obtained via the $v^{\prime}=1$ band, that shown in Figure 4 displays the greatest fraction $(\sim 208)$ of photoelectrons with $v^{+} \neq v^{\prime}$. At other wavelengths, $\mathrm{N}_{2}^{+} A{ }^{2} \Pi_{u^{\prime}} v^{+}=1$ can be produced with $>908$ purity. It is clear that the dominant ionization process correspond to excitation of the Rydberg electron, with preservation of the rctronic and vibrational state of the ion core. In addition, a lar fraction of those ions produced by core-switching transitions undesgo a change only in vibrational state, while retaining the $A{ }^{2} \pi_{u}$ ion core.

An extensive analysis of the vioronic structure in the region of the $\mathrm{N}_{2}$ spectrum containing the $\mathrm{O}_{3}{ }^{1} \mathrm{II}^{\prime} \mathrm{u}^{\prime}=1,2^{+}{ }^{+} \mathrm{X}{ }^{1}{ }^{\prime}{ }^{+} \mathrm{g}^{\prime}, v^{\prime \prime}=0$ bands has been reported recently by stahel et al. 18 This region is extremely complicated due to the mixing of three different ${ }^{1} \Pi_{u}$ states (see Figure 5), and due to the presence of three ${ }^{1} \Sigma_{u}^{+}$states that are alse strongly mixed. In the analysis of stahel et al. ${ }^{18}$, the $o_{3}{ }^{1} I_{u} V^{\prime}=1,2$ vibronic levels are found to contain 8.48 and $10.08 \mathrm{~b}{ }^{1} \mathrm{IT}_{\mathrm{u}}$ character, respectively, and somewhat less $c^{1} \mathrm{I}_{\mathrm{u}}$ 


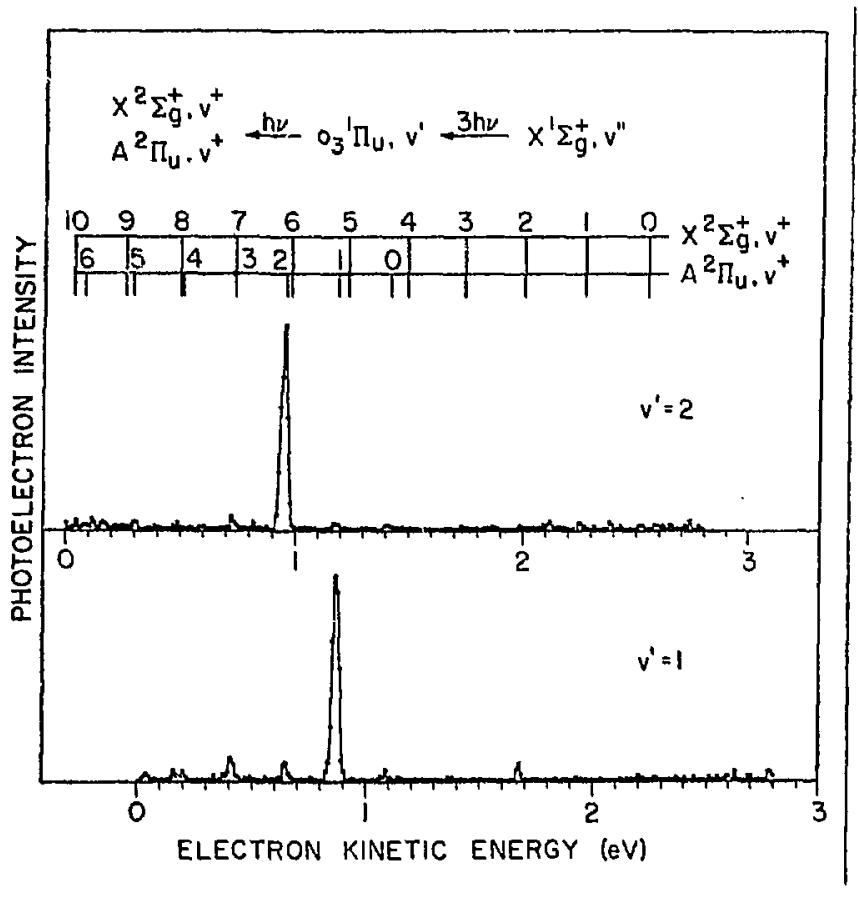

Figure 4. Photoelectron spectra of $\mathrm{N}_{2}$ determined following (3+1) multiphoton ionization via the $o_{3}{ }^{1} \Pi_{u}$, $v^{\prime}=1,2+x^{1} \Sigma_{g^{\prime}}^{+3} v^{\prime \prime}=0$ bands.

character. In addition, a rotational analysis by Yoshino et al, 19 of the single photon absorption data for the $\mathrm{O}_{3}{ }^{1} I_{\mathrm{u}}+\mathrm{x} \mathrm{i}_{\mathrm{V}}^{+}$ transition indicates much stronger mixing at the R-branch bandhead of the $v^{\prime}=1$ band due to $a$ perturbation by the $b^{1} I_{u^{\prime}} v^{\prime}=12$ level. one would expect these perturbations to strongly affect the vibrational branching ratios following multiphoton ionization. In particular, photoionization of high vibrational levels of the $b{ }^{1} I_{4}$ state, which is a valence state, would be expected to populate a wide distribution of $\mathrm{N}_{2}^{+}$vibrational levels in both the $x^{2} \Sigma_{g}^{+}$and A $2 \mathrm{~J}_{\mathrm{u}}$ states. It is clear from Figure 4 that this is not the case. Calculations of Michel.s 20 indicate that two different electron configurations are important in describing the $b{ }^{1} \pi_{4}$ state of $\mathrm{N}_{2}$. Both of these configurations, $\ldots\left(2 \sigma_{u}\right)^{2}\left(1 \pi_{u}\right)^{3}\left(3 \sigma_{g}\right)^{1}\left(1 \pi_{q}\right)^{2}$ and $\ldots\left(2 \sigma_{u}\right)^{1}\left(1 \pi_{u}\right)^{4}\left(3 \sigma_{g}\right)^{2}\left(1 \pi_{g}\right)^{1}$, differ from the $x^{2} \Sigma_{g}^{+}$ard $A{ }^{9} \pi_{u}$ states of $\mathrm{N}_{2}$ by two orbitals. For this reason the photoicnization cross section for the $b{ }^{1} \Pi_{u}$ state may be relatively small., leading to a dominance of the $\mathrm{O}_{3}{ }^{1} \mathrm{II}_{\mathrm{u}}$ character in the photoionizaition of the intermediate state. Similar behavior in the photoelectron spectra obtained following $(3+1)$ nultiphoton ionization of $\mathrm{N}_{2}$ via the $\mathrm{b}^{1} \mathrm{II}_{\mathrm{u}}$ ' $v^{\prime}=3-5$ and the $c^{1} \pi_{u^{\prime}} v^{\prime}=0$ bands has been observed and is discussed below.

PHOTOIONIZATION OF THE $b{ }^{1} \pi_{u}$ AND $c^{1} \pi_{u}$ STATES OF $N_{2}$

In preliminary studies of $(3+1)$ multiphoton ionization of selected vibrational levels of the $b^{1} \pi_{u}$ and $c{ }^{1} \Pi_{u}$ states of $N_{2}$, we 


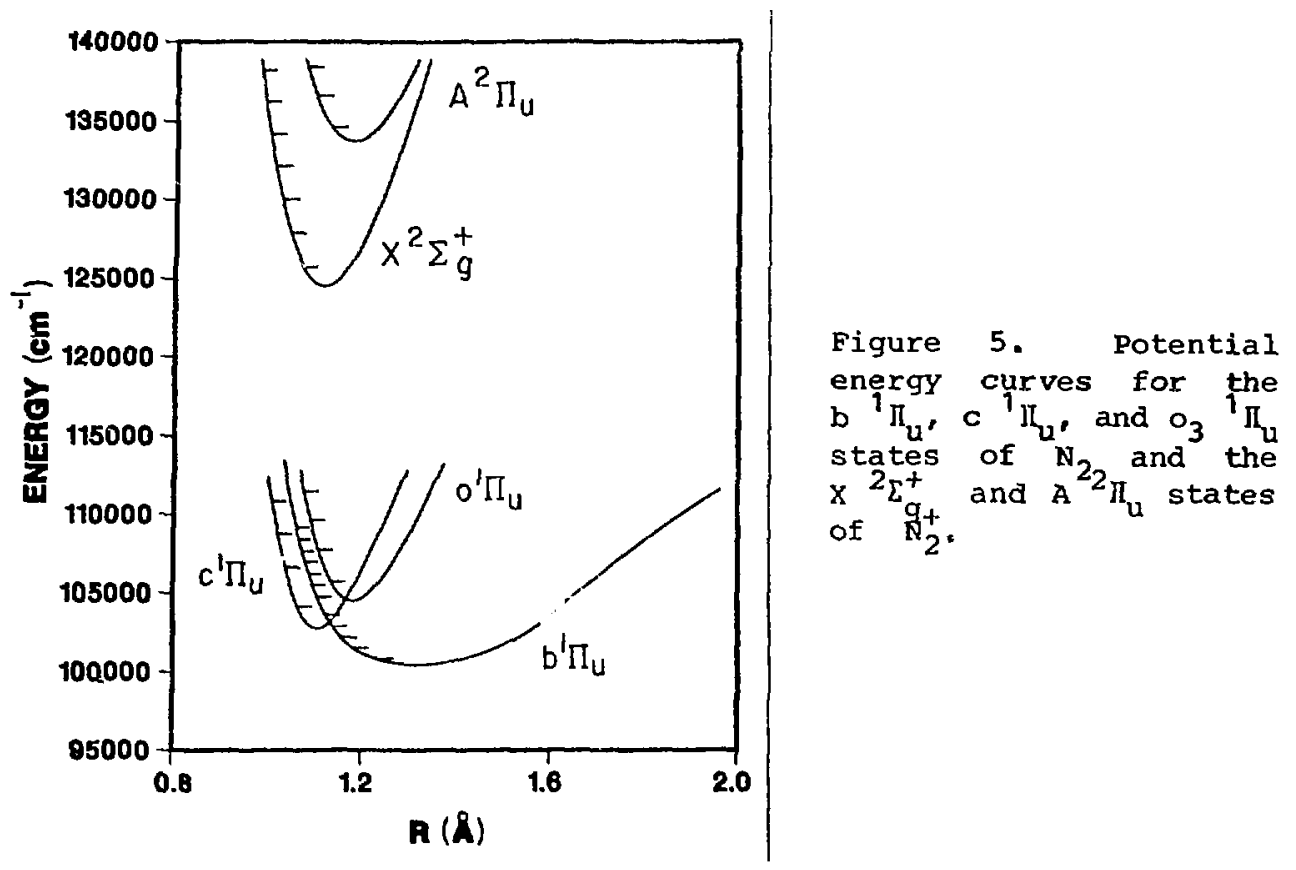

see further evidence of the effect of Rydberg-valence mixing on the photoelectron spectra. Figure 6 shows photoelectron spectra recorded at the wavelengths of the three photon transitions to the $k$-branch bandheads of the $b{ }^{1} \Pi_{u^{\prime}}, v^{\prime}=3-5+x^{1} \Sigma_{g^{\prime}}^{+} v^{\prime \prime}=0$ and $c^{1} \Pi_{u}$, $v^{\prime}=0+x^{1} \Sigma_{g^{\prime}}^{+} v^{\prime \prime}=0$ bands. The analysis of the vibronic structure of these states reported by stahel et al. ${ }^{18}$ shows that these levels are strongly mixed. The percentage vibronic character of each of these levels is given in Table II for the three main components of each wavefunction. (Note that the effects of local rotational perturbations, which are quite severe in some of these states, are not included in this analysis. ${ }^{21}$ ) The data of Table II show that each of these levels is a complex and unique mixture of $c^{1}{ }^{1}, \quad v=0$ and $b^{\prime}{ }^{\prime} \Pi_{1}, v=2-5$, with smaller components (less than 10\%) of other levels. In spite of this, all of the photoelectron spectra shown in Figure 6 look remarkably similar, displaying one dominant peak at the $\mathrm{N}_{2}^{+}, \mathrm{v}^{+}=0$ level. This is suggestive of ionization via a Rydberg state with a ground state core converging to $\mathrm{v}^{+}=0$, i.e. the $c^{1}{ }^{1} \mathrm{u}_{\mathrm{u}}$, $v^{\prime}=0$ level. It would therefore appear that the photoionization cross section from the $b^{1} \Pi_{u}$ electronic component of these levels is relatively small. However, while this interpretation appears to explain the results shown in Figure 6 , it is less satisfactory for other bands in this region that are currently under investigation. 22 It is clear that more work is needed to fully understand these results. In particular, more complete and systematic cudies are needed, which would include photoelectron angular distributions and photoelectron spectra determined as a function of the rotational level of the resonant intermediate state. 


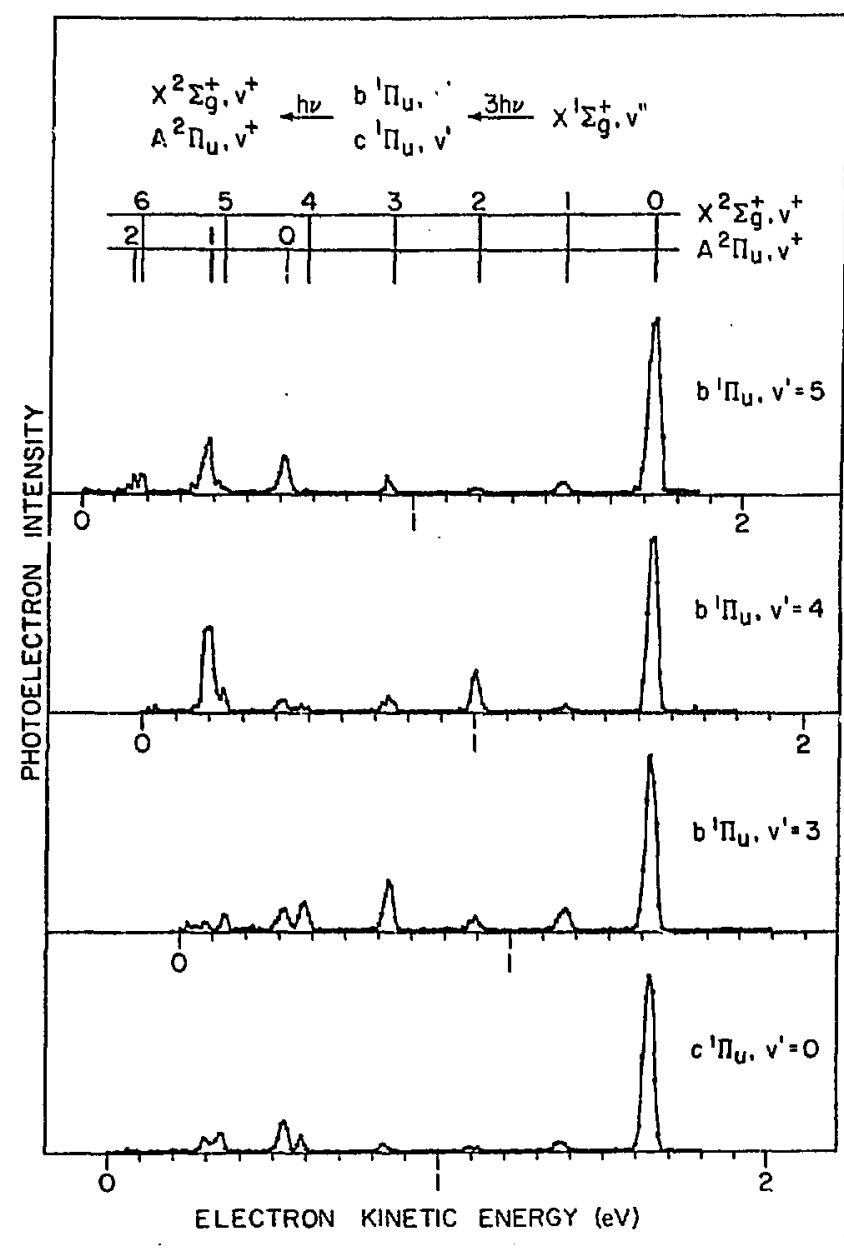

Figure 6. Photoelectron spectra of $\mathrm{N}_{2}$ determined following $(3+1)$ multiphoton ionization via tire $b{ }^{1} \Pi_{j}^{\prime}, v^{\prime}=3-$ $5+x^{1} \Sigma_{g^{\prime}}^{+} \quad v^{\prime \prime}=0$ and the $c 1 \mathrm{I}^{\prime}, \quad v^{\prime}=0$ + $x^{1} \Sigma_{g}^{+} v^{\prime}=0$ bands.

Table II. Percentage vibronic Character for tre $c^{1} I_{U^{\prime}} v=0$ and $b^{1} \Pi_{u}, v=3-5$ Levels of $\mathrm{N}_{2}$

Le'vel

co $\quad$ b2

b3

b4

b5

$\infty$

other

$c^{1} \pi_{a}, v=0 \quad 33.6$

$30.3 \quad 24.0$

12.1

$b^{1} \Pi_{u}, v=3$

5.8

8.4

75.7

10.1

$b^{1} \mathrm{~L}_{\mathrm{u}}, \mathrm{v}=4$

16.0

14.459 .3

10.3

$b^{1} \Pi_{a}, v=5$

22.1

64.0

4.4

9.5

(a) Data from Reference 18. The column headings are appreviations of the appropriate term symbols, e.g. $b 2$ is $b{ }^{9} I_{u}, v=2$. 
With the ability to prepare rotational and vibrational stateselected excited molecular states and to photoionize these states with a single photon, the field of molecular photoionization dynamics enters a new stage of development. Thu present studies represent some of the early attempts to exploit the technique of resonantly enhanced multiphoton ionization to gain information on the excited states of small molecules, which are theoretically tractable. Future developments in the study of the dynamics of excited state molecular photoionization include (work in some of these areas already is being performed by various groups): (1) determination of the photoelectron angular distributions following multiphoton ionization; $2 e, 23$ (2) MPI/PES studies in which the excited state is prepared using one wavelength and then is ionized using an independently variable wavelength; (3) MPI/PES studies of autoionizing Rydberg states; and (4) MPI/PES studies of excited state photodissociation products.

Another aspect of resonant multiphoton ionization is that it allows the selective ionization of one component of a complex mixture. Thus, state preparation of lons and the study of their subsequent reactions could be performed in the same region of space. This would allow the study of reactions of ions in relatively short-lived electronic states without interference from extraneous ionization products. However, the present studies illustrate that one cannot rely, a priori, on the Frarck-condon principle to determine the vibrational state distributions following resonantly enhanced multiphoton ionization. Until these processes are better understood, state-selected studies should be performed only after photoelectron energy analysis has confirmed the actual state distribution.

\section{REFERENCES}

1. See, for example, P. M. Johnson and C. E. Otis, Ann. Rev. Phys. Chem. 32, 139 (1981).

2. For examples of MPI/PES studies of small molecules, see (a) J. C. Miller and R. N. Compton, J. Chem. Phys. 75, 22 (1981); (b) M. G. White, M. Seaver, W. A. Chupka, and S. D. Colson, Phys. Rev. Lett. 49, 28 (1982); (c) J. Kimman, P. Kruit, and M. J. van der wiel, Chem. Phys. Lett. 88, 576 (1982); (d) J. H. Glownia, S. J. Riley, S. D. Colson, J. C. Miller, and R. N. Compton, J. Chem. Phys. 77, 68 (1982); (e) Y. Achiba, K. Sato, K. Shobatake, and K. Kimura, J. Chem. Phys. 78, 5474 (1983); (f) S. R. Long, J. T. Meek, and J. P. Reilly, J. Chem. Phys. 79, 3206 (1983); and S. T. Pratt, P. M. Dehmer, and J. L. Dehmer, J. Chem. Phys. 79, $3234(1983)$. Also, for a review of MPI/PES processes in atoms and molecules to early 1983, see K. Kimura, Adv. Chem. Phys. (in press).

3. S. T. Pratt, E. D. Poliakoff, P. M. Dehmer, and J. I. Dehmer, J. Chem. Phys. 78, 65 (1983). 
4. S. T. pratt, P. M. Dehmer, and J. I. Dehiner, J. Chem. Phys. 78, 4315 (1983).

5. S. T. Eratt, P. M. Dehmer, and J. I. Dehmer, Chem. Phys. Lett. (in press).

6. S. T. Pratt, P. M. Dehmer, and J. I. Dehmer, J. Chern. Phys. (in press).

7. G. Hexzberg, Molecular Spectra and Molecular structure II. Spectra of Diatomic Molecules (Van Nostrand Reinhold, Princeton, 1950).

8. J. B. Halpern, H. Zacharias, and R. Wallenstein, J. Mol. Spectrosc. 79, 1 (1980).

9. U. Fans and D. Dill, Phys. Rev. A 6, 185 (1972).

10. D. Dill, Rhys. Rev. A 6, 160 (1972).

11. D. Dill, in photoionization and Other Probes of Many-Electron Interactions, edited by F. J. Wuilleumier (Plenun, New York, 1976), p. 387 .

12. H. M. Crosswhite, The Hydrogen Molecule Wavelength Tables of Heinrich Dieke (Wiley-Interscience, New York, 1972).

13. K. P. Huber and G. Herzberg, Molecular Spectra and Molecular Structure IV. Constants of Diatomic Molecules (Van Nostrand Reinhold Company, New York, 1979).

14. I. Dabrowski and G. Herzberg, Can. J. Phys. 66, 5584 (1977).

15. Franck-Condon factors were calculated using the numerical potential of W. Kolos and J. Rychlewski, J. Mol. Spectrosc. 62, 109 (1976) for the $C{ }^{1} I_{u}$ state and that of $H$. Wind, $J$. Chem. Phys. 42, 2371 (1965) with the adiabatic corrections of $D$. $M$. Bishop and R. W. Wetmore, Mol. Phys. 26, 145 (1973) for the $\mathrm{X}^{2} \mathrm{\Sigma}_{\mathrm{g}}^{+}$ionic state.

16. A. Loftus and P. H. Krupenie, J. Phys. Chen. Ref. Data 6, 113 (1977).

17. (a) J. Ganz, B. Lewandowski, A. Siegel, W. Bussert, H. fiaibel, M. W. Ruf, and H. Hotop, J. Phys, B. 15, L485 (1982); (b) A. Siegel, J. Ganz, W. Bussert, and H. Hotop, J. Phys. B 16, 2945 (983); and (c) K. Sato, Y. Achiba, and K. Kimura, J. Chem. Phys. 80, 57 (1984). See also R. F. Stebbings, F. B. Dunning, and R. D. Rundel, in Atomic Physics Volume 4, edited by G. zu Putlitz,

E. W. Weber, and A. Winnacker (Plenum, New York, 1975), p. 713, and references therein.

18. D. Stahel, M. Ieoni, and K. Dressler, J. Chem. Phys. 79, 2541 (1983).

19. K. Yoshino, Y. Tanaka, P. K. Carroll, and P. Mitchell, J. Mol. Spectrosc. 54, 87 (1975).

20. H. H. Michels, Adv. Chem. Phys. 45, 225 (1981).

21. P. K. Carroll and C. P. Collins, Can. J. Phys. 47, 553 (1969).

22. S. T. Pratt, P. M. Dehmer, and J. I. Dehmer, (to be published).

23. (a) M. G. White, W. A. Chupka, M. Seaver, A. Woodward, and S. D. Colson, J. Chem. Phys. 80, 678 (1983); and (b) S. L. Anderson, G. D. Kubiak, and R. N. Zare, Chem. Phys. Lett. (in press). 